\title{
EFFECT OF CHAIR AEROBICS ON QUALITY OF LIFE IN SEDENTARY OBESE INDIVIDUALS
}

\author{
Soniya T. Lohana, ${ }^{1, B, C, D}$ Trupti Yadav², A \\ ${ }^{1}$ Intern, Faculty of physiotherapy, Krishna institute of medical sciences "Deemed to be" University, Karad, Maharashtra, India \\ ${ }^{2}$ Assisstant Professor, Department of Musculoskeletal sciences, Faculty of Physiotherapy, Krishna Institute of Medical Sciences \\ "Deemed to be" University, Karad, Maharashtra, India

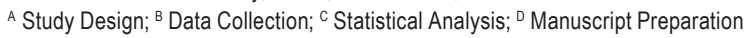

\section{Address for correspondence:} \\ Trupti Yadav \\ Faculty of Physiotherapy, Krishna Institute of Medical Sciences "Deemed to Be" University, Karad, Maharashtra, India \\ E-mail: drtruptiwarude@gmail.com
}

\begin{abstract}
Ahstract Background: A sedentary lifestyle is associated with various negative health outcomes, including obesity. Aerobic exercise is shown to have beneficial effects on all body systems. The purpose of the present study was to demonstrate the effect of chair aerobics as a light to moderate intensity exercise on the quality of life of obese sedentary individuals.

Objectives: Objectives of the study were to improve the physical function and the quality of life in obese individuals in a sedentary working environment in order to improve the energy levels and reduce fatigue.

Material and methods: An experimental study was conducted among sedentary obese individuals. Subjects were screened pre-intervention using OSPAQ, BMI etc. Chair aerobics was given in the form of group therapy as a light to moderate intensity exercise programme. Quality of life was assessed post intervention by assessing self esteem and activity levels and physical functioning through QLQ II and SF 36 questionnaires.

Results: The study showed statistically significant differences in BMI $(p<0.0001)$, self esteem and activity levels $(p<0.0001)$, energy levels and general health $(p<0.0001)$.

Conclusion: Chair aerobics showed a significant effect on the quality of life of sedentary obese individuals with improved levels of physical functioning, increased energy levels and reduced fatigue levels.
\end{abstract}

Key worlds chair aerobics, sedentary, obesity, physical activity, workplace

\section{Introduction}

Sedentary behaviour is defined as "any waking behaviour characterized by an energy expenditure less than a 1.5 metabolic equivalent of task (MET) while in a sitting or reclining posture" (Straker, Coenen, Dustan, Gilson, Healy, 2016). The metabolic equivalent of task (MET) is the energy expended for an activity and is defined as the ratio of the energy expended during quiet sitting (Straker et al., 2016).

Sedentary behaviour thus refers to a distinct class of behaviours either performed while sitting or reclining with low energy expenditure (Straker et al., 2016). 
Sedentary behaviour is conceptually different to "physical inactivity", which is defined as "performing insufficient amounts of moderate to vigorous intensity physical activity, not meeting the specified physical activity guidelines" (Straker et al., 2016). Moderate intensity physical activity is defined as $>3$ to $<6 \mathrm{METs}$, and vigorous intensity activity as $>6$ METs. Light activity is $>1.5$ to $<3 \mathrm{METs}$. A broad range of behaviours fall within this band.

Sedentary lifestyle has been shown to be detrimentally associated with degenerative cardiometabolic outcomes (such as cardiovascular diseases, diabetes and obesity), musculoskeletal problems, mental ill-health, and poor quality of life.

A sedentary lifestyle is also associated with an increase in BMI over time, thus leading to obesity (Straker et al., 2016). Reducing sedentary behaviours could help prevent an increase in BMI and thereby reduce the occurrence of obesity (Mitchell et al., 2014). Physical activity has been shown to reduce stress and anxiety traits and to improve physical self-perception and mental well-being ("Effect of aerobic fitness on the physiological stress responses at work - PubMed-NCBI," n.d.). Aerobic fitness also helps to reduce stress responses and improves an individual's capacity for coping with stress ("Effect of aerobic fitness on the physiological stress responses at work. - PubMed-NCBI," n.d.). Chair Aerobics are aerobic exercises performed while sitting in a chair, which include upper and lower body movements led by an instructor while listening to music and sitting in a straight-back chair (Robinson, Masud, Hawley-Hague, 2016). Chair based exercises have been shown to have a beneficial effect at maintaining or promoting independence and mobility in older people and also in patients post CABG (Anthony, n.d.; Thapa, Pattanshetty, 2016). Compliance to chair based programs is generally better than that of standing or dynamic exercise, especially amongst low baseline levels of fitness and function. Chair based exercise has specific benefits as a training method as it stabilizes the lower spine by providing a fixed base, and it facilitates a greater range of movement by providing points of leverage and support; it minimizes load-bearing and reduces balance problems in those with particularly poor mobility (Robinson et al., 2016).

In certain professions, physical activity levels at work are low while sedentary behaviour is high. This largely depends on the function of the occupation (white-collar vs. blue-collar). A sedentary lifestyle leads to various diseases and disorders that may lead to deleterious health outcomes of the patients. A sedentary lifestyle is also associated with reduced quality of work and increased stress levels in individuals, affecting their overall psychosocial well-being. A sedentary work environment is associated with reduced levels of physical fitness along with various musculoskeletal conditions and psychosocial stress. Chair aerobics is an alternative to dynamic exercises while working, for individuals with a sedentary work environment or sedentary lifestyle.

This study thus focuses on increasing the levels of physical fitness, improving the quality of life and reducing the stress and anxiety levels in individuals with a sedentary nature of work. Previous research focuses on various issues related to sedentary work behaviour and thus studies which will address the decrease in quality of work due to sedentary behaviour are required.

\section{Methodls}

This study was a pre/post experimental design with a selection of employees working in an institute, randomly selecting employees based on inclusion and exclusion criteria. The data was collected from individuals or employees with a sedentary work environment i.e. sitting for $>3 \mathrm{~h} / \mathrm{d}$ (according to OSPAQ) during the study period or extending for 6 months. Ethical approval for the study was granted by the Institutional Ethical Committee. Both genders aged 25-45 willing to participate in the study were considered for selection. An informed consent form was filled in by 
the participants. Those with a BMI more than 30 and those who were unable to do weight bearing exercises were selected.

Subjects having a history of any psychological conditions, congenital or genetic disorders or with major abdominal or cardiovascular surgeries were excluded from the study. Statistically analysis was done using "InStat" for Windows v3.06. Intra group comparisons were done by applying a "Paired t-test" to the pre- and post-treatment values of the group for the BMI and SF-36 Questionnaire.

A Wilcoxon matched paired test was used on intra group analysis of non-parametric data (Moorehead-Ardelt Quality of Life Questionnaire II) (Moorehead, Ardelt-Gattinger, Lechner, Oria, 2003). Probability value $p<0.05$ was considered statistically significant.

Procedure:

a) subjects with a sedentary working environment were screened and selected on the basis of inclusion criteria;

b) 53 Subjects were screened by assessing BMI, Occupational Sitting and Physical Activity Questionnaire (OSPAQ), and 36 subjects fulfilled the criteria;

c) sedentary participants were selected on the basis of OSPAQ, at >3hr/day due to their nature of work. QLQII and SF36 Questionnaire were filled in by these subjects;

d) informed consent was taken from the participants. Each was explained the procedure of the study;

e) warm-up exercises were given in the form of an active Range of Movement (AROM) for the Upper Limb and Lower Limb followed by a session of Chair Aerobics. A gradual increase in intensity and frequency was done according to the FITT principle. (Frequency, Intensity, Time, Type). The exercise session ended with stretching and relaxation (diaphragmatic breathing exercises) as a cool-down phase;

f) each exercise was given for 30-45 min for 4 days/week over a period of 8 weeks;

g) chair aerobics was given as a group therapy to all the subjects. The following exercises were given:

- free neck exercises,

- shoulder and elbow rotations,

- wrist curls and rotations,

- ankle toe movements,

- spine flexion and extension,

- spinal side rotation;

h) initially, 5 sets of each exercise were given, gradually increasing it to 10 sets. Total time duration of warm up phase was 15 minutes;

i) exercises included in the aerobic session:

- marching with arm movement,

- alternate hand and leg movements,

- alternate arm and leg raise,

- knee to chest,

- v step,

- claps on head,

- marching in standing position,

- hamstring curls, 
- leg swings,

- lunges,

- knee to chest in a standing position;

j) initially, low intensity exercise was performed, gradually increasing the speed of movements and thus their intensity. Total time duration of chair aerobics was 30-40 minutes Exercises included in the cool-down session:

- neck stretch,

- forearm stretch,

- triceps stretch,

- pectoral stretch,

- quadriceps stretch,

- hamstring and ta stretch (the stretches were sustained from 10 seconds and up to 30 seconds. Total time duration of the cool down phase was 10-15 minutes);

k) 25 individuals participated throughout the study for 8 weeks. There were 11 dropouts due to the work and deadlines to be covered by these employees;

I) after the intended duration of treatment, the efficacy of the treatment protocol was observed;

m) the data collection sheets were filled in post-test and assessed;

n) statistical analysis was done with appropriate biostatistical tools.

\section{Results}

A total of 56 subjects with a sedentary lifestyle (OSPAQ $>3 \mathrm{hr} /$ day) were screened during the study recruitment period, out of which 36 were enrolled on the basis of inclusion criteria. 25 individuals completed the exercise training period. The results of the study were based on data collected for 25 subjects. The mean age of the subjects was $38 \pm 7.03$ years. The 25 subjects comprised 11 males and 14 females. Pre- and post-intervention outcomes were measured. There was a significant difference in BMI, at $p<0.0001$. Also there was significant difference in self esteem and activity levels assessed by Moorehead-Ardelt Quality of Life Questionnaire, and a significant improvement was seen in all components.

Self esteem and self-image assessed by "Usually I feel..." was changed from "very badly about myself" to "very good about myself" ( $p<0.0001)$. Interest in Physical activities developed from "not at all" to "very much" $(p<0.0001)$. Social contacts were increased probably due to the group exercises from none to some $(p=0.0313)$. Increased satisfaction with sex life was significant $(p=0.0039)$ and their outlook towards "between meal munching" (i.e. approach towards food) changed, and thus overeating was prevented after incorporating physical activity in their schedule $(p=0.0313)$.

Overall, the Self Esteem and Activity levels were considerably increased by including some form of physical activity in the sedentary working lifestyle of the obese individuals. Levels of Physical Functioning were improved significantly $(p=0.0006)$. Role Limitation due to Physical Health was reduced significantly $(p=0.0014)$. Role Limitation due to Emotional Health was reduced significantly $(p<0.000)$. Energy levels were improved and fatigue levels were reduced $(p<0.0001)$. Emotional Well Being of individuals was better $(p=0.0184)$ which is significant. Social Functioning was improved ( $p=0.0012)$. Body pain was considerably reduced $(p=0.001)$ and General Health of the individuals was better than the pre-test $(p<0.0001)$. 


\section{Discussion}

This study was conducted to evaluate the effect of chair aerobics on quality of life in sedentary obese individuals. A sedentary lifestyle is associated with various types of disorders. M. Castillo-Retamal and E.A. Hinckson (2011) suggest that now-a-days a sedentary activity level in the workplace is high due to a change in the nature of modern job functions, and thus physical activity in the workplace should be measured and intervention should be made to reduce the ill-effects of a sedentary lifestyle (a study by Jonathan A. Mitchell suggests that a sedentary lifestyle is associated with an increase in BMI over time and thus, an increase in BMI is associated with occurrence of various morbid health conditions).

Chair aerobics is a form of aerobic exercise performed while sitting in a chair or with the help of a chair, which includes rhythmic upper and lower body movements matching the beats of music. Chair aerobics is a low to medium intensity exercise in which the spine is stabilized by a fixed base of support compared to that of standing or dynamic exercises, which require a lot of stability (also chair aerobics facilitate a greater range of movement by providing points of leverage and support, it also minimizes load-bearing on joints while some postural muscles are relaxed; it also reduces balance problems in those with poor mobility and makes a great form of exercise while sitting).

The main aim of this study was to evaluate the effectiveness of chair aerobics on an improvement of quality of life of obese and sedentary individuals. The objectives of the study were to improve the physical fitness and quality of life in individuals with a sedentary working environment, to improve their energy levels by reducing fatigue levels in these individuals. 53 individuals participated in the study out of which 36 individuals fulfilled the selection criteria and 25 individuals continued their participation in the study for the 8 weeks. Subjects were screened by measuring their BMI and by measuring the hours they sit by Occupational Sitting and Physical Activity Questionnaire (OSPAQ) to rule out their sedentary nature of work. The 25 individuals comprised 11 males and 14 females. It was seen that the BMI of the females was higher than that of the males. A study entitled "Global Gender Disparities in Obesity: A Review" by Rebecca Kanter et al. supports this difference noted, by suggesting that in developing countries women are more likely to have a higher BMI than men.

The chair aerobics sessions were carried out 4 days per week for 8 weeks, when BMI was again measured. Along with BMI, QLQII and SF36 Questionnaire were also assessed. Outcome measures showed significant differences among BMI and the components of both the Questionnaires. This was confirmed using statistical analysis using a 'Paired t-test' and non-parametric Wilcoxon tests for intra group comparisons.

Within the intra group comparison, the pre-treatment BMI was $31.77 \pm 3.23$ and dropped to $30.31 \pm 3.19$ $(p<0.0001)$ which showed a small but significant decrease in BMI. Also, the Self esteem and Activity levels assessed by Moorehead-Ardelt Quality of Life Questionnaire, a significant improvement was seen in all components. Self esteem and Self-image, Interest in physical activities had improved. Social contacts increased probably due to the group exercises. Satisfaction from sex life increased and their outlook towards food as a way of providing nutrition increased. Thus, snacking between the meals was reduced and overeating was prevented. Overall. the Self Esteem and Activity levels were considerably better after including some form of physical activity in the sedentary working lifestyle of the obese individuals.

The levels of Physical Functioning improved significantly with $p=0.0006$. Role Limitation due to Physical Health and emotional health reduced significantly. Energy levels improved and fatigue levels reduced with $p<0.0001$. Emotional Well Being of individuals was better. Social Functioning improved. Body pain was considerably reduced and General Health of individuals was better than pre-intervention.Physical activity and exercise have a wide range 
of health benefits. Studies show that physical activity can boost self-esteem, mood, quality of sleep and energy, and reduce the risk of stress, anxiety and various other disorders. According to research, regular aerobic exercise appears to boost the size of the hippocampus, thereby improving verbal memory and learning along with numerous physiological effects seen in various systems of the body. A study entitled "Health benefits of physical activity: the evidence" by Darren E.R. Warburton, suggests that an increase in energy expenditure from physical activity of $1,000 \mathrm{kcal}(4,200 \mathrm{~kJ})$ per week or an increase in physical fitness of $1 \mathrm{MET}$ (metabolic equivalent) was associated with a mortality reduction of about $20 \%$. An increase in physical fitness will reduce the risk of pre-mature death, while modest enhancements in physical fitness in previously sedentary people have been associated with large improvements in health status. Thus, exercise has numerous benefits and chair aerobics is one such new type of low to medium energy impact exercise. It makes use of energy by incorporating low to high energy as needed by modifying the intensity of the exercise with maximum amount of benefit during working hours in individuals who cannot specifically incorporate exercise in their day to day life due to their work schedule.

Incorporating exercise in the workplace will also address the stress and anxiety issues faced by employees. Presently, there is a lack of exercise and physical activity in various professions due to the digital and sedentary nature of our work. Perhaps, incorporating chair aerobics as a group or by using ear-phones by an individual can serve to fill that void of physical activity and help to enhance the benefits of physical activity and exercise in the workplace.

While considering chair aerobics, there is an increase in cardiopulmonary responses as well as other physiological changes. Stress and anxiety are reduced, and self esteem, body image and self-confidence improve with the help of chair aerobics. Exercise has numerous benefits on overall health and thus this could be used by individuals while at work to make individuals healthy and fit in all domains of life and prevent diseases and disorders.

Although the study duration was short and limited to a small sample size, it can be stated that chair aerobics with a low to moderate intensity reduces the BMI of individuals and has a positive impact on overall general health. A future study with a large sample size or RCT comparing effect chair aerobics with different treatment forms or dietary advice can be undertaken.

\section{Conclusion}

This study concluded that there was a significant effect of chair aerobics on the quality of life of sedentary obese individuals, with improved levels of physical functioning, increased energy levels, and reduced fatigue levels with much more added health benefits.

\section{Funding}

This research received no external funding.

\section{Acknowledgments}

We would like to acknowledge the institution, head of the department, participants, peers, teachers and those who have guided us throughout the study period. 


\section{Conflicts of Interest}

The authors declare no conflicts of interest.

\section{References}

Anthony, K. (n.d.) (2013). Chair-Based Exercises for Frail Older People: A Systematic Review. BioMed Research International.

Berde, S.D., Yadav, T.S., Gosavi, P.M., Gijare, S.S. (2019). Effect of Core Strengthening Exercises \& Chair Aerobic Exercises in Primary Dysmenorrhoea. International Journal of Health Sciences, 3, 6 .

Castillo-Retamal, M., Hinckson, E.A. (2011). Measuring physical activity and sedentary behaviour at work: A review. Work (Reading, Mass.), 40 (4), 345-357. DOI: 10.3233/WOR-2011-1246.

Effect of aerobic fitness on the physiological stress responses at work. PubMed-NCBI. (n.d.). Retrieved from: https://www.ncbi.nlm. nih.gov/pubmed/17708014 (3.11.2019).

Health benefits of physical activity: The evidence. (n.d.). Retrieved from: https://www.ncbi.nlm.nih.gov/pmc/articles/PMC1402378/ (3.11.2019).

Kanter, R., Caballero, B. (2012). Global Gender Disparities in Obesity: A Review1. Advances in Nutrition, 3 (4), 491-498. DOI: 10.3945/ an.112.002063.

Mitchell, J.A., Bottai, M., Park, Y., Marshall, S.J., Moore, S.C., Matthews, C.E. (2014). A Prospective Study of Sedentary Behavior and Changes in the BMI Distribution. Medicine and Science in Sports and Exercise, 46 (12), 2244-2252. DOI: 10.1249/ MSS.0000000000000366.

Moorehead, M.K., Ardelt-Gattinger, E., Lechner, H., Oria, H.E. (2003). The validation of the Moorehead-Ardelt Quality of Life Questionnaire II. Obesity Surgery, 13 (5), 684-692. DOI: 10.1381/096089203322509237.

Psychosocial benefits of workplace physical exercise: Cluster randomized controlled trial | BMC Public Health | Full Text. (n.d.). Retrieved from: https://bmcpublichealth.biomedcentral.com/articles/10.1186/s12889-017-4728-3 (3.11.2019).

Robinson, K.R., Masud, T., Hawley-Hague, H. (2016). Instructors' Perceptions of Mostly Seated Exercise Classes: Exploring the Concept of Chair Based Exercise (Research article). DOI: 10.1155/2016/3241873.

Sedentary time and its association with risk for disease incidence, mortality, and hospitalization in adults: A systematic review and metaanalysis. PubMed-NCBI. (n.d.). Retrieved from: https://www.ncbi.nlm.nih.gov/pubmed/25599350 (3.11.2019).

Straker, L., Coenen, P., Dustan, D., Gilson, N., Healy, G. (2016). Sedentary Work - Evidence on an Emergent Work Health and Safety Issue.

Thapa, S., Pattanshetty, R. (2016). Effect of Chair aerobics as low intensity exercise training on heart rate, blood pressure and six minute walk distance in post coronary artery bypass graft surgery patients through phase I cardiac rehabilitation. Nepalese Heart Journal, 13, 19. DOI: 10.3126/njh.v13i1.14540.

Cite this article aS: Lohana, S.T., Yadav, T. (2020). Effect of Chair Aerobics on Quality of Life in Sedentary Obese Individuals. Central European Journal of Sport Sciences and Medicine, 1 (29), 21-27. DOI: 10.18276/cej.2020.1-03. 